\title{
The Uniform Asymptotic Stability of MMC under Open Loop Operation
}

\author{
Chaoying $\mathrm{Xia}^{1}$ and Jiali $\mathrm{Yu}^{1}$ \\ ${ }^{1}$ School of Electrical and Information Engineering, Tianjin University, Tianjin, China
}

\begin{abstract}
With the wide application of modular multilevel converters (MMC) in high voltage and high power areas, many open loop methods are proposed for MMC. However, the stability of MMC open loop control system has not been solved so far. Aim at this problem, on the base of Lyapunov theory and persistently and sufficiently exciting (PSE) condition in the adaptive control theory, the uniform asymptotic stability of MMC with capacitor voltage open-loop and without current closed-loop is proved. For the capacitor voltage open-loop balancing control, a soft-rotating phase-shifted carrier (PSC) modulation algorithm is used. Considering the influence of nonideal factors such as the deviation of capacitance and power tubes' characteristics, the correctness and validity of these results are verified by the simulation.
\end{abstract}

Keywords-Modular Multilevel Converter (MMC); Lyapunov theory; persistently and sufficiently exciting (PSE); uniform asymptotic stability

\section{INTRODUCTION}

MMC has its certain advantages of modularity, scalability, and low harmonic distortion, in comparison with conventional two-level converters, diode-clamped converters and cascaded H-bridge converters [1]. Therefore, MMC has been regarded as the preferred topology in the high-voltage high-power applications [2-4].

After the concept of MMC was put forward, the capacitor voltage balancing strategy is always the most special and important aspect, since it is theoretically and practically significant to the system performance, reliability and complexity. The majority of strategies are based on either the nearest level modulation (NLM) combined with a capacitor voltage sorting algorithm [5] or the pulse width modulation (PWM) method with capacitor voltage feedback control [6] These balancing methods are mainly based on capacitor voltage measurements. As a result, the reliability of control system may be deteriorated as the number of SMs increases.

To solve the afore mentioned problem, in terms of topology, the literature [7] proposed a new parallel-connected diodeclamped MMC. In this topology, the capacitor voltages can be balanced automatically without any balancing control algorithms. However, the balancing speed is slow and the circuit is complex. In terms of algorithms, the commonly used method is to design a capacitor voltage balancing method by observer [8-10]. In [11] and [12], a balancing technique without using capacitor voltage feedback control is proposed which depends on rotating an optimal pulse pattern obtained offline. The open-loop rotating method reduces the complexity of the control system, and the need for capacitor voltage measurements is reduced to fault-handling purposes or increase system safety performance. However, the stability problem of capacitor voltage open-loop operation has not been solved. Many researchers might assume that the capacitor voltage open-loop control will be less robustness against transients and non-ideal factors [13]. In this paper, it will be shown that when the switching functions of SMs satisfy some certain conditions, the capacitor voltages in one arm will be balanced even though there is no capacitor voltage closed-loop control. And for capacitor voltages balancing control, the capacitor voltage detection and observation are not necessary.

\section{BASIC OPERATION PRINCIPLES OF MMC}

Fig.1 schematically illustrates the circuit of a gridconnected $\mathrm{MMC}$ for HVDC application. The topology comprises a three-phase MMC, a YN $\Delta$ type transformer and an AC system. Each phase of the MMC is made up by an upper arm and a lower arm. Each arm consists of $N$ SMs and an arm inductor. Each SM contains a half-bridge circuit and a capacitor. There are three basic operating modes for each SM, including charging, discharging and bypassed. A control strategy continuously determines the number of inserted SMs of each arm to obtain a multilevel voltage at the AC side of MMC. Meanwhile, a SM selection method chooses which SM in each arm to insert or bypass to balance the capacitor voltage.

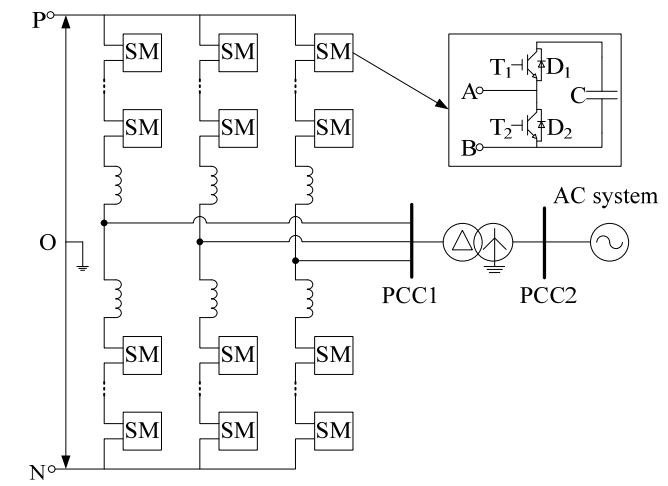

FIGURE I. THE CIRCUIT CONFIGURATION OF MMC-HVDC INVERTER STATION

\section{MODELING OF MMC}

In the majority of literatures published about the modeling of MMC, it is generally assumed that the capacitor voltages 
have been balanced by means of the sorting method or feedback control $[14,15]$. But established models cannot describe the charging and discharging dynamics of each SM and they are invalid for the analysis of capacitor voltages without measurements or feedback control.

In this paper, the stability of MMC open loop control systems will be investigated. In which, the capacitor voltages are neither detected nor observed, so no feedback control or sorting control will be adopted. The non-ideal factors, such as the differences of the capacitances and the power tubes' characteristics of SMs will be taken into account. Therefore, it is necessary to establish a full-dimensional model of MMC which takes each SM capacitor voltage as state variable and considers the asymmetrical parameters.

According to the topology of MMC in Fig.1, the equivalent circuit of the phase $\mathrm{x}(\mathrm{x}=\mathrm{a}, \mathrm{b}$ or $\mathrm{c})$ of $\mathrm{MMC}$ is depicted in Fig.2.

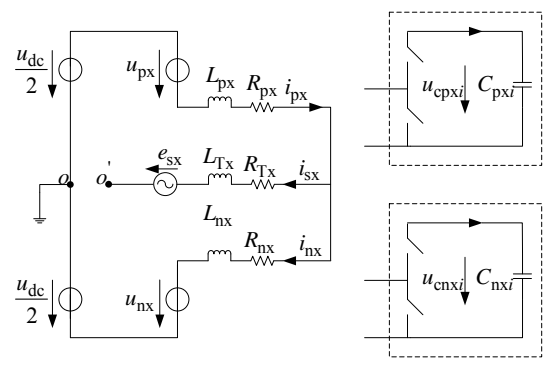

FIGURE II. THE EQUIVALENT CIRCUIT FOR X PHASE MMC

In Fig.2, the measured dc bus voltage $u_{\mathrm{dc}}$ is constant. The measured grid voltage $e_{\mathrm{sx}}$, has already been converted to the $\Delta$ side of the YN $\Delta$ type, varies independently with time and is unrelated to load condition. $L_{\mathrm{px}}$ and $L_{\mathrm{nx}}$ are referred to as inductances of upper and lower arms. Equavalent resistences in the two arms are denoted as $R_{\mathrm{px}}$ and $R_{\mathrm{nx}}$. The capacitace of the $\mathrm{i}$-th submodule in the upper and lower arms are $C_{\mathrm{pxi}}$ and $C_{\mathrm{nxi}}$ respectively. $u_{\mathrm{px}}$ and $u_{\mathrm{nx}}$ are the arm voltage of upper and lower arm. $i_{\mathrm{px}}$ and $i_{\mathrm{nx}}$ are arm currents of $\mathrm{x}$ phase. $i_{\mathrm{sx}}$ is output current of $\mathrm{x}$ phase at the ac side of MMC. Circulating current is defined as $i_{\mathrm{cx}}=\left(i_{\mathrm{px}}+i_{\mathrm{nx}}\right) / 2$.

According to Fig.2, by the Kirchhoff's voltage law, the system dynamic equations are given by

$$
\left\{\begin{array}{l}
L_{\mathrm{px}} \frac{\mathrm{d}}{\mathrm{d} t} i_{\mathrm{px}}=-R_{\mathrm{px}} i_{\mathrm{px}}+\frac{u_{\mathrm{dc}}}{2}-u_{\mathrm{sx}}-\sum_{i=1}^{N} s_{\mathrm{px} i} u_{\mathrm{cpx} i} \\
L_{\mathrm{nx}} \frac{\mathrm{d}}{\mathrm{d} t} i_{\mathrm{nx}}=-R_{\mathrm{nx}} i_{\mathrm{nx}}+\frac{u_{\mathrm{dc}}}{2}+u_{\mathrm{sx}}-\sum_{i=1}^{N} s_{\mathrm{nx} i} u_{\mathrm{cn} x i}
\end{array}\right.
$$

Where, $u_{\mathrm{sx}}=L_{\mathrm{Tx}} \frac{\mathrm{d}}{\mathrm{d} t} i_{\mathrm{sx}}+R_{\mathrm{Tx}} i_{\mathrm{sx}}+e_{\mathrm{sx}}+u_{\mathrm{o}^{\prime} \mathrm{o}}$, in which $u_{\mathrm{o}^{\prime} \mathrm{o}}$ is the voltage between the midpoint ' $O$ ', of the ac side and the midpoint ' $O$ ' of the de side.

The capacitor voltage dynamics can be expressed by

$$
\left\{\begin{array}{l}
\frac{\mathrm{d}}{\mathrm{d} t} u_{\mathrm{cpxi}}=\frac{s_{\mathrm{px} i}}{C_{\mathrm{cpxi}}} i_{\mathrm{px}} \\
\frac{\mathrm{d}}{\mathrm{d} t} u_{\mathrm{cnxi}}=\frac{s_{\mathrm{nxi}}}{C_{\mathrm{cnxi}}} i_{\mathrm{nx}}
\end{array}\right.
$$

Considering the constraint of $i_{\mathrm{sa}}+i_{\mathrm{sb}}+i_{\mathrm{sc}}=0$, and substituting the arm currents $i_{\mathrm{px}}=i_{\mathrm{cx}}+1 / 2 i_{\mathrm{sx}}$ and $i_{\mathrm{nx}}=i_{\mathrm{cx}}-1 / 2 i_{\mathrm{sx}}$ into equations (1) and (2). Then taking the circulating currents of phase ' $a$ ', ' $b$ ' and 'c' and the ac-side currents of phase ' $a$ ' and ' $b$ ' (namely $\boldsymbol{x}_{1}$ ) and the $6 \mathrm{~N}$ capacitor voltages (namely $\boldsymbol{x}_{2}$ ) as the state variables, the dc bus voltage and the grid voltages (namely $\boldsymbol{e}=\left[\begin{array}{llll}u_{\mathrm{dc}} & u_{\mathrm{sa}} & u_{\mathrm{sb}} & u_{\mathrm{sc}}\end{array}\right]^{\mathrm{T}}$ ) as the inputs, the state-space description of the three-phase MMC is derived as

$$
\dot{\boldsymbol{x}}=\left[\begin{array}{cc}
A & -B S\left(s_{\mathrm{pxi}}, S_{\mathrm{nxi}}\right) \\
M S^{\mathrm{T}}\left(s_{\mathrm{pxi}}, s_{\mathrm{nxi}}\right) & 0
\end{array}\right] \boldsymbol{x}+\left[\begin{array}{c}
B H \\
0
\end{array}\right] \boldsymbol{e}(3)
$$

Where, the state variables are $\boldsymbol{x}^{\mathrm{T}}=\left[\begin{array}{ll}\boldsymbol{x}_{1}^{\mathrm{T}} & \boldsymbol{x}_{2}{ }^{\mathrm{T}}\end{array}\right]$. The matrix $H \in R^{5 \times 4}$ is expressed as

$$
H=\left[\begin{array}{ccccc}
1 & 0 & 1 & 0 & 1 \\
0 & -1 & 0 & 0 & 0 \\
0 & 0 & 0 & -1 & 0 \\
0 & 1 & 0 & 1 & 0
\end{array}\right]^{\mathrm{T}}
$$

The matrix $M \in R^{6 N \times 6 N}$ includes the capacitances of $6 N$ SMs, which is expressed as

$$
M=\operatorname{diag}\left[\begin{array}{lll}
1 / C_{\mathrm{pal}} & \cdots & 1 / C_{\mathrm{ncN}}
\end{array}\right]
$$

The matrix $A=B D$, in which $B \in R^{5 \times 5}$ and $D \in R^{5 \times 5}$ are indicated by 


$$
B=\left[\begin{array}{ccccc}
L_{\mathrm{pa}}+L_{\mathrm{na}} & \left(L_{\mathrm{pa}}-L_{\mathrm{na}}\right) / 2 & 0 & 0 & 0 \\
\left(L_{\mathrm{pa}}-L_{\mathrm{na}}\right) / 2 & {\left[\begin{array}{c}
\left(L_{\mathrm{pa}}+L_{\mathrm{na}}+L_{\mathrm{pc}}+L_{\mathrm{nc}}\right) / 4 \\
+L_{\mathrm{Ta}}+L_{\mathrm{Tc}}
\end{array}\right]} & 0 & \left(L_{\mathrm{pc}}+L_{\mathrm{nc}}\right) / 4+L_{\mathrm{Tc}} & -\left(L_{\mathrm{pc}}-L_{\mathrm{nc}}\right) / 2 \\
0 & 0 & L_{\mathrm{pb}}+L_{\mathrm{nb}} & \left(L_{\mathrm{pb}}-L_{\mathrm{nb}}\right) / 2 & 0 \\
0 & \left(L_{\mathrm{pc}}+L_{\mathrm{nc}}\right) / 4+L_{\mathrm{Tc}} & \left(L_{\mathrm{pb}}-L_{\mathrm{nb}}\right) / 2 & {\left[\begin{array}{c}
\left(L_{\mathrm{pb}}+L_{\mathrm{nb}}+L_{\mathrm{pc}}+L_{\mathrm{nc}}\right) / 4 \\
+L_{\mathrm{Tb}}+L_{\mathrm{Tc}}
\end{array}\right]} & -\left(L_{\mathrm{pc}}-L_{\mathrm{nc}}\right) / 2 \\
0 & -\left(L_{\mathrm{pc}}-L_{\mathrm{nc}}\right) / 2 & 0 & -\left(L_{\mathrm{pc}}-L_{\mathrm{nc}}\right) / 2 & L_{\mathrm{pc}}+L_{\mathrm{nc}}
\end{array}\right]^{-1}
$$

The matrix $S\left(s_{\mathrm{pxi}}, S_{\mathrm{nxi}}\right) \in R^{5 \times 6 N}$ contains $6 N$ switching functions, that is

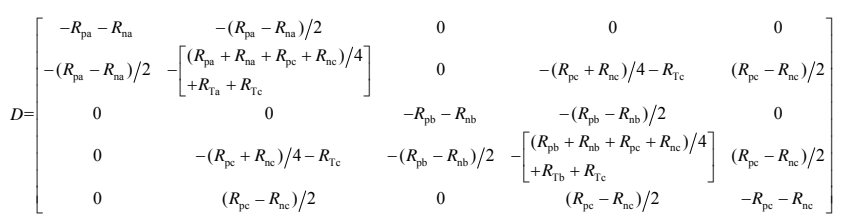

$\left(R_{\mathrm{pc}}-R_{\mathrm{nc}}\right) / 2$

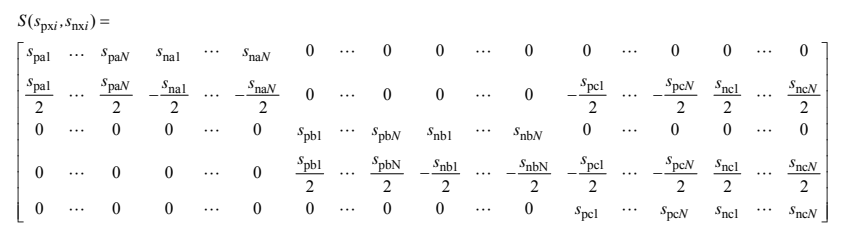

\section{STABILITY OF MMC}

It is commonly doubted that the different power tubes' characteristics or capacitances of SMs may cause the continuous deviation of capacitor voltages under the openloop control. The aim of the capacitor voltage balancing can only be achieved by the feedback control of capacitor voltages which are either detected or observed. The following contents, based on the established model of MMC and by the Lyapunov theory and the adaptive control theory, it will show that the capacitor voltages will be balanced under the open-loop control as long as some certain condition is satisfied.

For the capacitor voltages open-loop control without current closed-loop, the switching function of each SM varies independently over time and is independent of state variables. The system given in equation (3) is linear time-varying. As is known, the stability of a linear system is equivalent to its corresponding non-excitated system. The non-excitated system of the equation (3) can be expressed as,

$$
\dot{\boldsymbol{x}}=\left[\begin{array}{cc}
A & -B S\left(s_{\mathrm{pxi}}(t), s_{\mathrm{nxi}}(t)\right) \\
M S^{\mathrm{T}}\left(s_{\mathrm{pxi}}(t), s_{\mathrm{nxi}}(t)\right) & 0
\end{array}\right] \boldsymbol{x}
$$

Let a Lyapunov function candidate be $\mathrm{V}=1 / 2 \boldsymbol{x}_{1}^{\mathrm{T}} B^{-1} \boldsymbol{x}_{1}+1 / 2 \boldsymbol{x}_{2}^{\mathrm{T}} M^{-1} \boldsymbol{x}_{2}$. According to the equation (4), it is easily to verify that $\dot{V} \leq 0$, then the zero-state responses of system (4) are uniformly stable. Furthermore, the uniform asymptotic stability for the system (4) can be achieved by the conclusion which is associated with the model reference adaptive control system. Taking the state transition $\mathbf{Z}=T \boldsymbol{x}$, where the matrix $T$ is defined as,

$$
T=\left[\begin{array}{cc}
I & 0 \\
0 & M^{-1 / 2}
\end{array}\right]
$$

$I \in R^{5 \times 5}$ is an identity matrix. The equation (1) is equivalent to

$$
\left[\begin{array}{c}
\dot{\mathbf{z}}_{1} \\
\dot{\mathbf{z}}_{2}
\end{array}\right]=\left[\begin{array}{cc}
A & -B S\left(s_{\mathrm{pxi}}(t), s_{\mathrm{nxi}}(t)\right) M^{1 / 2} \\
M^{1 / 2} S^{\mathrm{T}}\left(s_{\mathrm{pxi}}(t), s_{\mathrm{nxi}}(t)\right) B^{\mathrm{T}} P & 0
\end{array}\right]\left[\begin{array}{l}
\mathbf{z}_{1} \\
\mathbf{z}_{2}
\end{array}\right]
$$

Let $P=B^{-1}$, then the matrix $P A+A^{\mathrm{T}} P$ is negative. According to the literature, the zero trivial solution of system (4) is uniformly asymptoticly stable if and only if there exist positive numbers $T_{0}, \varepsilon_{0}$ and $\delta_{0}$ such that if $w \in R^{m}$ is a unit vector, then there is a sequence $t_{n} \rightarrow \infty$ with $t_{n+1}-t_{n} \leq T_{0}$ and for all $n$,

$$
\varepsilon_{0} \leq\left\|\int_{t_{n}}^{t_{n}+\delta_{0}} B S\left(s_{\mathrm{pxi}}(t), s_{\mathrm{nxi}}(t)\right) M^{1 / 2} w \mathrm{~d} t\right\|
$$

The condition given by inequality (7) is the PSE condition of $B S\left(s_{\mathrm{pxi}}(t), s_{\mathrm{nxi}}(t)\right) M^{1 / 2}$, that is, the spectrums of switching functions should be rich enough. Then a necessary condition of inequality (7) is that

$$
\varepsilon_{0} \leq \int_{t_{n}}^{t_{n}+\delta_{0}}\left\|B S\left(s_{\mathrm{pxi}}(t), s_{\mathrm{nxi}}(t)\right) M^{1 / 2} w\right\| \mathrm{d} t
$$

For the simplified analysis, supposing the parameters of $\mathrm{MMC}$ are symmetrical. In this case, by an elementary line transformation $F$, the matrix $B S\left(s_{\mathrm{pxi}}(t), s_{\mathrm{nxi}}(t)\right) M^{1 / 2}$ is simplified and transformed to

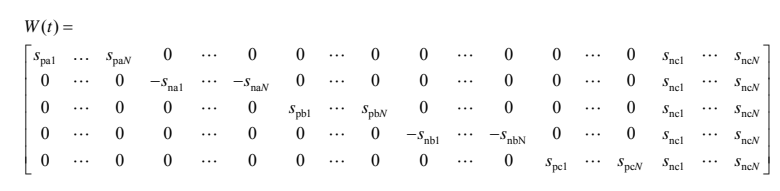

Then, 


$$
\begin{aligned}
& \int_{t_{n}}^{t_{n}+\delta_{0}}\left\|F B S\left(s_{\mathrm{pxi}}(t), s_{\mathrm{nxi}}(t)\right) M^{1 / 2} w\right\| \mathrm{d} t \\
& =\int_{t_{n}}^{t_{n}+\delta_{0}}\|W w\| \mathrm{d} t \leq\|F\| \int_{t_{n}}^{t_{n}+\delta_{0}}\left\|B S\left(s_{\mathrm{pxi}}(t), s_{\mathrm{nxi}}(t)\right) M^{1 / 2} \boldsymbol{w}\right\| \mathrm{d} t
\end{aligned}
$$

As the matrix $F$ is nonsingular, if $W$ satisfies the PSE condition, that is, there exists a positive $\varepsilon$,

$$
\varepsilon \leq \int_{t_{n}}^{t_{n}+\delta_{0}}\|W w\| \mathrm{d} t
$$

Then the matrix $B_{2} S_{2}\left(s_{\mathrm{pxi}}(t), S_{\mathrm{nxi}}(t)\right) M^{1 / 2}$ will satisfy the PSE condition.

To verify the the uniform asymptotic stability of MMC under open loop operation, a soft-rotating Phase-Shifted Carrier (PSC) modulation method is used, which requires that the phase-difference between each carrier and modulation signal should rotate. In this paper, the carrier frequency of the soft-rotating PSC modulation is set to $312.5 \mathrm{~Hz}$. The parameters of the simulation are given as followings: the DC bus voltage is $5 \mathrm{kV}$, the number of SMs per arm is 4 , the arm inductance is $0.265 \mathrm{mH}$, the arm resistance is $0.03 \Omega$, the capacitance of each SM is $4000^{\mu \mathrm{F}}$, the converted peak grid voltage is $2.3 \mathrm{kV}$ and $50 \mathrm{~Hz}$, the converted resistance and inductance at $\mathrm{AC}$ side are $0.065 \Omega$ and $2.01 \mathrm{mH}$.

Fig. 3 shows the waveforms of capacitor voltages of the upper and lower arms of phase ' $a$ ' under zero-input, that is, the DC bus voltage and the grid voltages are set to zero, and the initial values of capacitor voltages of the upper arm of phase 'a' are set to $800 \mathrm{~V}, 1200 \mathrm{~V}, 1400 \mathrm{~V}$ and $1600 \mathrm{~V}$, the initial values of other SMs are entirely set to 1250V. As displayed in Fig.3, the zero-input responses of capacitor voltages will decay to zero as $t \rightarrow \infty$ and they are independent of their initial state values. The uniformly asymptotically stable result of MMC is verified.
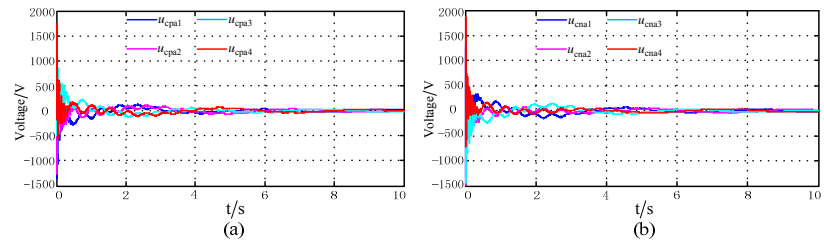

FIGURE III. THE WAVEFORMS OF CAPACITOR VOLTAGES OF PHASE 'A' IN UPPER ARM (A) AND LOWER ARM (B)

Moreover, to verify the capacitor voltage balancing performance and robustness under open-loop condition, the inputs of the DC bus voltage and grid voltages are applied and soft-rotating PSC modulation method is used. Firstly, to verify the balancing ability when a load step changes, the command of the AC-side active power is changed from $1.5 \mathrm{MW}$ to $0.75 \mathrm{MW}$ when $\mathrm{t}=5 \mathrm{~s}$ and the reactive power is always set to 0 Var. To verify the balancing robustness when non-ideal factors exist, one is that the capacitances of SMs are set to different values, in which the capacitances of SMs of the upper arm of phase ' $\mathrm{a}$ ' are set to $4400 \mu \mathrm{F}, 4200 \mu \mathrm{F}$, $3800 \mu \mathrm{F}$ and $3600 \mu \mathrm{F}$, the capacitances of the other SMs are entirely set to $4000 \mu \mathrm{F}$. The other is the conduction-drops of SMs are set to different values. The conduction-resistances of power tubes of the upper arm of phase 'a' are set to $1 \mathrm{~m} \Omega, 3$ $\mathrm{m} \Omega, 5 \mathrm{~m} \Omega$ and $8 \mathrm{~m} \Omega$, the conduction-resistances of the other power tubes are entirely set to $0.01 \mathrm{~m} \Omega$. Fig.4 shows the waveforms of capacitor voltages of the upper and lower arms of phase ' $a$ ' in the three experiments designed above, where the initial capacitor voltage of each SM is the same as Fig.3. The active power and reactive power at the AC-side under the load step changes are shown in Figure 5.
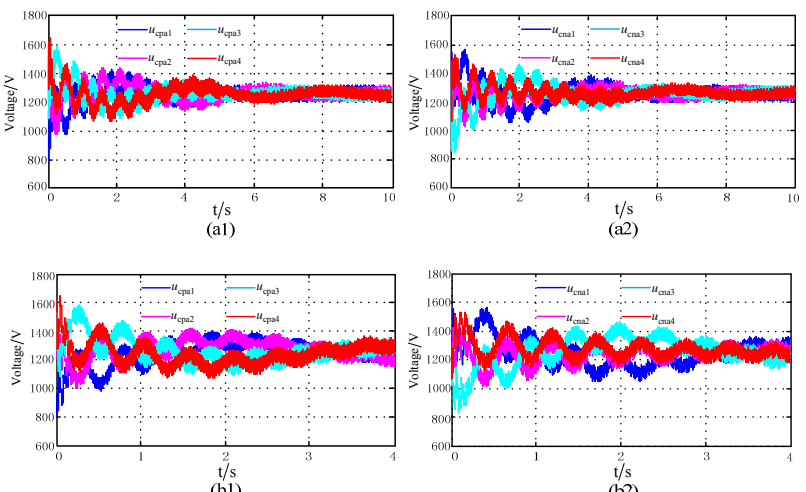

(b2)
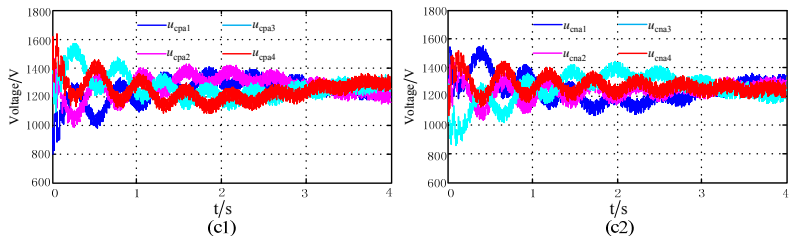

FIGURE IV. THE CAPACITOR VOLTAGES OF PHASE 'A' IN UPPER ARM (A1) AND LOWER ARM (A2) WHEN A LOAD STEP CHANGES

THE CAPACITOR VOLTAGES IN UPPER ARM (B1) AND LOWER ARM (B2) WHEN THE DIFFERENCES OF CAPACITANCES EXIST

THE CAPACITOR VOLTAGES IN UPPER ARM (C1) AND LOWER ARM (C2) WHEN THE DIFFERENCES OF CONDUCTION-DROPS EXIST.
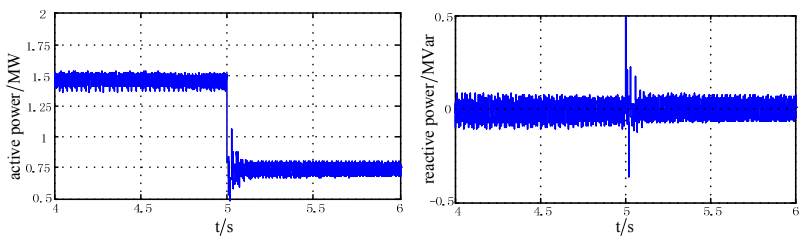

FIGURE V. THE ACTIVE POWER AND REACTIVE POWER WHEN A LOAD STEP CHANGES

\section{CONCLUSION}

In this paper, the necessary and sufficient condition for uniformly asymptotic stability of MMC open loop control systems are obtained by the Lyapunov theory and the adaptive 
control theory. To verify the theory, the soft rotating PSC modulation algorithm is used. The simulation results show that the capacitor voltage can be balanced without any form of feedback and is not sensitive the no ideal factors. This conclusion will change the design idea of $\mathrm{MMC}$, which has an important theoretical and practical significance.

\section{REFERENCES}

[1] R L A M. An Innovative Modular Multilevel Converter Topology Suitable for a Wide Power Range[C]. IEEE Bolongna PowerTech Conference, Bologna, Italy, 2003.

[2] Picas R, Ceballos S, Pou J, et al. Closed-loop discontinuous modulation technique for capacitor voltage ripples and switching losses reduction in modular multilevel converters [J]. IEEE Transactions on Power Electronics, 2015, 30(9): 4714-4725.

[3] Adam G P, Williams B W. Half-and full-bridge modular multilevel converter models for simulations of full-scale HVDC links and multiterminal DC grids [J]. IEEE Journal of Emerging and Selected Topics in Power Electronics, 2014, 2(4): 1089-1108.

[4] Debnath S, Qin J, Bahrani B, et al. Operation, control, and applications of the modular multilevel converter: A review [J]. IEEE transactions on power electronics, 2015, 30(1): 37-53.

[5] Yu F, Lin W, Wang X, et al. Fast voltage-balancing control and fast numerical simulation model for the modular multilevel converter [J]. IEEE Transactions on Power Delivery, 2015, 30(1): 220-228.

[6] Sasongko F, Akagi H. Low-Switching-Frequency Operation of a Modular Multilevel DSCC Converter with Phase-Shifted RotatingCarrier PWM [J]. IEEE Transactions on Power Electronics, 2016.

[7] Gao C, Lv J. A New Parallel-Connected Diode-Clamped Modular Multilevel Converter With Voltage Self-Balancing[J]. IEEE Transactions on Power Delivery, 2017, 32(3):1616-1625.

[8] Picas R, Zaragoza J, Pou J, et al. New measuring technique for reducing the number of voltage sensors in modular multilevel converters [J]. IEEE Transactions on Power Electronics, 2016, 31(1): 177-187.

[9] D'Arco S, Suul J A. Estimation of sub-module capacitor voltages in modular multilevel converters[C]//Power Electronics and Applications (EPE), 2013 15th European Conference on. IEEE, 2013: 1-10.

[10] Abdelsalam M, Marei M, Tennakoon S, et al. Capacitor voltage balancing strategy based on sub-module capacitor voltage estimation for modular multilevel converters [J]. CSEE Journal of Power and Energy Systems, 2016, 2(1): 65-73.

[11] K. Ilves, A. Antonopoulos, S. Norrga, and H. P. Nee. A new modulation method for the modular multilevel converter allowing fundamental switching frequency [J]. IEEE Trans. Power Electron., vol. 27, no. 8,pp. 3482-3494, Aug. 2012.

[12] Ahmed K H, Adam G P. New modified staircase modulation and capacitor balancing strategy of 21-level modular multilevel converter for HVDC transmission systems [C]//Power Electronics, Machines and Drives (PEMD 2014), 7th IET International Conference on. IET, 2014: $1-6$.

[13] Huber J E, Korn A J. Optimized pulse pattern modulation for modular multilevel converter high-speed drive[C]//Power Electronics and Motion Control Conference (EPE/PEMC), 2012 15th International. IEEE, 2012: LS1a-1.4-1-LS1a-1.4-7.

[14] Jovcic D, Jamshidifar A A. Phasor model of modular multilevel converter with circulating current suppression Control [J]. IEEE Transactions on Power Delivery, 2015, 30(4): 1889-1897.

[15] Vatani M, Bahrani B, Saeedifard M, et al. Indirect finite control set model predictive control of modular multilevel converters [J]. IEEE Transactions on Smart Grid, 2015, 6(3): 1520-1529.

[16] Morgan A P, Narendra K S. Asymptotic stability of nonautonomous equations and the adaptive observer[C]//Decision and Control including the 15th Symposium on Adaptive Processes, 1976 IEEE Conference on. IEEE, 1976, 15: 1228-1230.

[17] L. Angquist, A. Antonopoulos, D. Siemaszko, K. Ilves, and M Vasiladiotis, "Open-Loop Control of Modular Multilevel Converters
Using Estimation of Stored Energy," IEEE Transactions on Industry applications, vol. 47, pp. 2516-2524, 2011. 